\title{
Markus Putnings
}

\section{Datenmanagement}

Abstract: Das (Forschungs-)Datenmanagement zeichnet sich durch eine systematische Planung und Durchführung von entsprechenden datenbezogenen Aufgaben unter Berücksichtigung von relevanten Kontextfaktoren rechtlicher, ethischer etc. Natur aus. Im Gegensatz zur populären Lebenszyklusmetapher können dabei Abläufe ineinander übergehen, nichtsequentiell ablaufen oder, je nach Art und Qualität der Daten, auch gänzlich wegfallen.

\section{Begriffsabgrenzung und Scope}

Das komplette Praxishandbuch widmet sich im Grunde dem (Forschungs-)Datenmanagement. Während die Schwerpunkte der vorherigen Abschnitte zwar auf den Kontextfaktoren des Datenökosystems, des jeweiligen Datenmarktes und der institutions-, fach- oder personenspezifischen Datenkultur legen, beeinflussen diese dennoch maßgeblich das individuelle (Forschungs-)Datenmanagement (s. Abb. 1). „Datenmanagement“ bedeutet in unserer Interpretation stets ein planvolles, methodisches Vorgehen von Individuen, Gruppierungen (z. B. Arbeits-, Projektgruppen) und Institutionen, im Gegensatz zu einer unreflektierten Behandlung von Daten:

Daten, Informationen und Wissen sind wertvolle Ressourcen, die geplant, organisiert und verwertet werden müssen. Im Umfeld eines Unternehmens spricht man hier allgemein von Managementfunktionen. ${ }^{1}$

Dieses Management (d.h. Planungs- und Verwaltungshandeln) gilt jedoch nicht nur für den Industrie- und Wirtschaftssektor, sondern sektorübergreifend auch für den Wissenschaftssektor, öffentlichen Sektor sowie die Bürgerwissenschaft. ${ }^{2}$ Oftmals werden diese Managementfunktionen und -aufgaben anhand einer Lebenszyklusmetapher angeordnet und beschrieben:

Daten, Informationen und Wissen können entstehen und vergehen, sie durchleben einen Zyklus, in dem sie erzeugt, gespeichert, auf verschiedene Arten benutzt, weitergegeben und auch wieder entfernt werden. ${ }^{3}$

1 Bodendorf 2006, 2.

2 S. Beitrag von Putnings, Kap. 1 in diesem Praxishandbuch.

3 Bodendorf 2006, 2. 


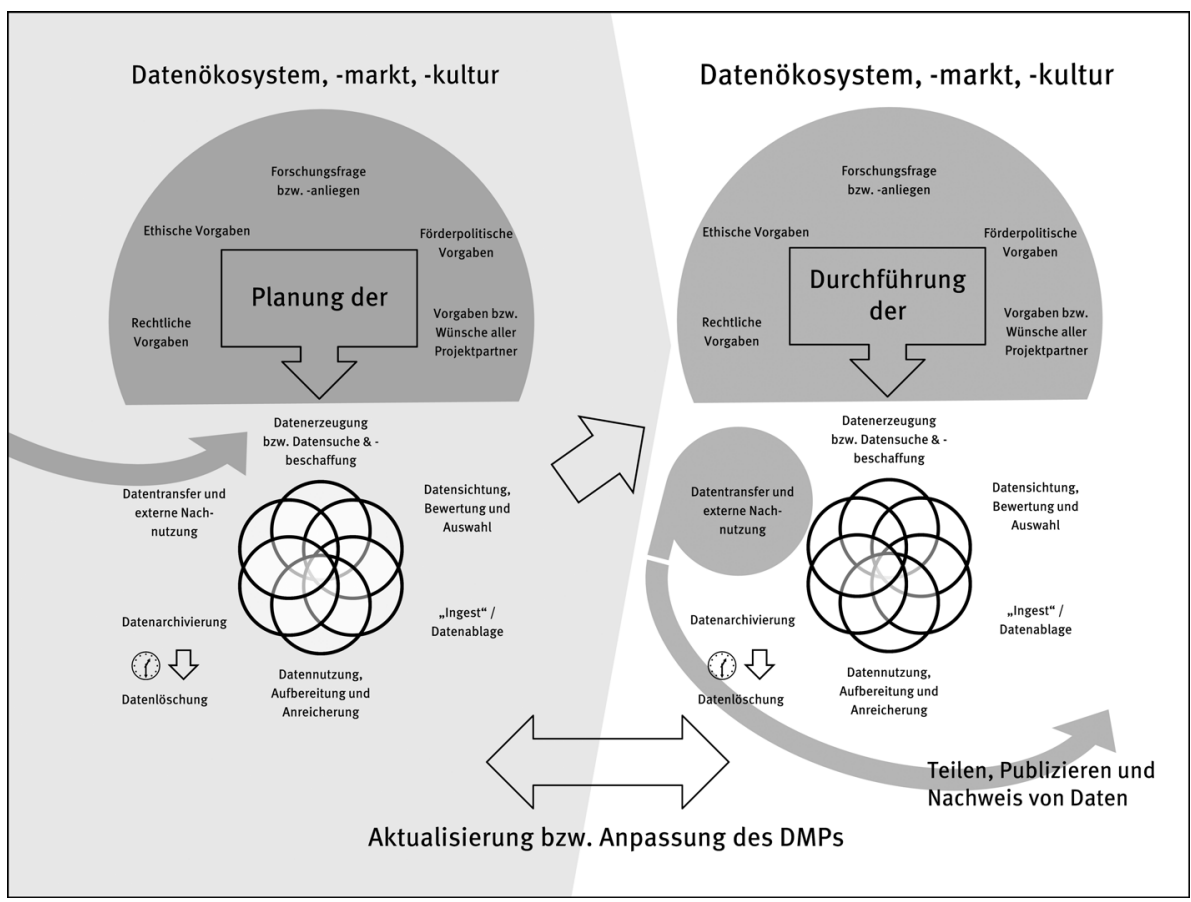

Abb. 1: Datenmanagement - Planung und Durchführung.

Die Metapher fand primär durch das populäre DCC Curation Lifecycle Model $^{4}$ Verbreitung. Es gibt in Teilen jedoch Kritik an der Dominanz der Lebenszyklusmetapher. ${ }^{5}$ Die Struktur des Praxisbuches betont einige Aspekte, die in der Fachliteratur teils etwas kurz kommen:

- Die Bedeutung nicht nur der Durchführung, sondern auch der bewussten Vorabplanung der Datenmanagementaufgaben, sei es mit oder ohne Datenmanagementplan (DMP), ${ }^{6}$

- die zu berücksichtigenden Kontextfaktoren aus Datenökosystem, -markt und -kultur und

- der nichtsequentielle bzw. teils sich verzweigende Ablauf, mit Möglichkeiten des partiellen Teilens, Publizierens, Veränderns und Löschens von Daten.

4 S. https://www.dcc.ac.uk/about/digital-curation. Letztes Abrufdatum der Internet-Dokumente ist der 15.11.2020.

5 S. z. B. https://www.zbw-mediatalk.eu/de/2018/09/forschungsdatenmanagement-abhaengigkeitvon-der-lebenszyklusmetapher-und-alternativen/.

6 Wobei ein DMP natürlich in vielen Fällen, vor allem bei datenintensiven Projekten, zu empfehlen wäre. 


\section{Planung und Durchführung des Datenmanage- ments}

Es gilt, sich in der Praxis jeweils konkret klar zu machen, was im Kontext von Daten geplant und organisiert werden muss. ${ }^{7}$ Im Rahmen von Förderanträgen können z. B. teils Kosten für die Aufbereitung, Archivierung, die Publikation und die (Nach-)Nutzung von Daten in Repositorien und Datenmärkten beantragt werden; ${ }^{8}$ entsprechend muss sehr frühzeitig, noch beim Forschungsdesign ${ }^{9}$ und beim Schreiben von Projektanträgen, an solche Punkte gedacht und diese eingeplant sowie ggf. in einem DMP schriftlich fixiert werden.

In Abhängigkeit vom jeweiligen Datenökosystem sollten zudem unter anderem (bundes-)länderspezifische Datenschutzregelungen, die Einbeziehung institutioneller Datenschutzbeauftragter, fachspezifischer Ethikkommissionen etc. berücksichtigt werden sowie, wenn später ein konkreter Datenmarkt bedient werden soll, dessen Anforderungsspezifikation. ${ }^{10}$

Wie die ineinandergreifenden Kreise in Abb. 1 verdeutlichen sollen, sind die Managementaufgaben teils miteinander verbunden, ergänzen sich gegenseitig oder fallen, je nach Daten(-qualität) teils auch gänzlich weg. ${ }^{11}$ Planung und Durchführung sind dabei eng verzahnt, da Projekte stetigen Veränderungsprozessen unterworfen sind. Manche Förderer, wie der Schweizerische Nationalfonds zur Förderung der wissenschaftlichen Forschung (SNF) fordern deshalb, den DMP eines geförderten Projekts zu festgelegten Zeitpunkten zu aktualisieren. ${ }^{12}$

7 Vgl. z. B. Netscher und Jensen 2019 für einen sehr detaillierten, praxisnahen Überblick über die systematische Planung und Umsetzung eines Datenmanagements im fachspezifischen Kontext der Sozialwissenschaften.

8 S. z. B. Beitrag von Putnings, Kap. 1.3 in diesem Praxishandbuch.

9 Hier können zusätzlich auch mögliche Synergieeffekte, spätere Weiterverwertbarkeiten etc. mitbedacht werden, vgl. Büttner, Hobohm und Müller 2011, 16.

10 Z. B. die Beachtung der Vokabulare für DCAT-AP.de im öffentlichen Sektor, s. https://www.dcatap.de/, https://www.govdata.de/standardisierung und der Beitrag von Schieferdecker, Kap. 2.3 in diesem Praxishandbuch.

11 Als konkrete Beispiele: wenn die Bewertung z. B. klar zeigt, dass alle Daten speicherungswürdig sind, erfolgt unter Umständen später keine entsprechende Selektion zur Archivierung. Bei Sichtung personenbezogener Informationen könnten jedoch im Rahmen einer speziellen Aufbereitung die Datensätze anonymisiert bzw. pseudonymisiert oder Löschfristen vorgesehen werden müssen. Teils entfällt, z. B. bei besonders sensiblen Daten, auch die Datenpublikation.

12 S. Beitrag von Putnings, Kap. 1.3 in diesem Praxishandbuch. 
Neben Vorgaben gibt es zur Unterstützung diverse Hilfsmittel bzw. Empfehlungen von Förderern zu einem bestmöglichen Datenmanagement. ${ }^{13}$ Auch einige Initiativen engagieren sich hierfür. ${ }^{14}$

\section{Fazit}

Wie gezeigt (s. Abb. 1), ist die Planung von Forschungsdaten sowie des entsprechenden Managements von hoher Bedeutung. ${ }^{15}$ Bei der anschließenden Durchführung des (Forschungs-)Datenmanagements liegen maßgebliche Schwerpunkte

- auf der Datenqualität (entsprechende Sichtung, Bewertung, Auswahl, nötige Aufbereitung und Anreicherung etc. $)^{16}$ und

- auf der Datenablage, der entsprechenden -kuration sowie finaler Langzeitarchivierung. ${ }^{17}$

Die zu beachtenden Einflussfaktoren auf die Planung und Durchführung sind dabei vielfältig und können z. B. dem Datenökosystem, Datenmarkt oder auch der Datenkultur entstammen. ${ }^{18}$ Zudem können für das Datenmanagement diverse Softwares sowie Ergebnisse und Erkenntnisse aus bestehenden Projekten und Initiativen nachgenutzt werden. ${ }^{19}$ Das Datenmanagement umfasst auch die Beschäftigung mit möglichen Datentransfers (z.B. Teilen, Publizieren) und mit der Nachnutzung von Daten über das Projektende hinaus und dient damit wiederum der potentiellen Suchmöglichkeit sowie leichteren Datenbeschaffung Dritter (s. Abb. 1, gesonderte Pfeile). ${ }^{20}$

13 S. Beitrag von Putnings, Kap. 1.3, sowie von Iglezakis und Hermann, Kap. 4.4 in diesem Praxishandbuch.

14 Vgl. z. B. https://bausteine-fdm.de/index, https://www.forschungsdaten.info/, https://www.forschungsdaten.org/index.php/Kategorie:Data_Management.

15 S. Beitrag von Dierkes, Kap. 4.1 in diesem Praxishandbuch.

16 S. Beitrag von Király und Brase, Kap. 4.3 in diesem Praxishandbuch.

17 S. Beitrag von Weber und Piesche, Kap. 4.2 in diesem Praxishandbuch.

18 S. Kap. 1 bis 3 in diesem Praxishandbuch.

19 S. Beitrag von Iglezakis und Hermann, Kap. 4.4 in diesem Praxishandbuch.

20 Da beides jedoch, bei einer „reinen“ Archivierung (z. B. bei besonders sensiblen Daten) entfallen kann, wird dies in Kap. 5 im Praxishandbuch gesondert behandelt. 


\section{Literatur}

Letztes Abrufdatum der Internet-Dokumente ist der 15.11.2020.

Bodendorf, Freimut. 2006. Daten- und Wissensmanagement. 2., aktualisierte und erweiterte Auflage. Springer-Lehrbuch. Berlin, Heidelberg: Springer. doi:10.1007/3-540-28682-9.

Büttner, Stephan, Hans-Christoph Hobohm und Lars Müller. 2011. „Research Data Management.“ In Handbuch Forschungsdatenmanagement, hg. v. Stephan Büttner, Hans-Christoph Hobohm und Lars Müller, 13-24. Bad Honnef: Bock u. Herchen. https://opus4.kobv.de/opus4-fhpotsdam/files/192/1.1_Research_Data_Management.pdf.

Netscher, Sebastian und Uwe Jensen. 2019. „Forschungsdatenmanagement systematisch planen und umsetzen. “ In Forschungsdatenmanagement sozialwissenschaftlicher Umfragedaten, hg. v. Uwe Jensen, Sebastian Netscher und Katrin Weller, 37-55. Leverkusen-Opladen: Barbara Budrich. doi:10.3224/84742233.04. 
